\title{
ASSESSMENT OF PHYSICIANS' COLORECTAL CANCER SCREENING PRIORITIES USING THE ANALYTIC HIERARCHY PROCESS (AHP)
}

\author{
James G. Dolan, MD * \\ Department of Community and Preventive Medicine \\ University of Rochester \\ Rochester, NY, USA \\ E-mail: James_Dolan@urmc.rochester.edu \\ Thomas F. Imperiale, MD \\ Department of Medicine \\ Indiana University School of Medicine \\ Indianapolis, IN, USA \\ E-mail: timperia@iupui.edu \\ Jeroan Allison, MD \\ Department of Medicine \\ University of Massachusetts Medical School \\ Worcester, MA, USA \\ E-mail: Jeroan.Allison@umassmed.edu \\ Emily Boohaker, MD \\ Department of Medicine \\ University of Alabama at Birmingham \\ Birmingham, AL, USA \\ E-mail: eboohake@uab.edu
}

\begin{abstract}
Introduction: Current colorectal cancer (CRC) screening guidelines endorse several options and recommend that patients and providers make choices through a shared decision making process. A key component of shared decision-making is determining decision priorities. Our goal was to assess primary care physicians' (PCP) priorities regarding currently recommended CRC screening programs.

Methods: PCPs from two geographically distinct sites completed an Analytic Hierarchy Process (AHP) analysis of ten CRC screening options for a typical, average-risk 50-year-old patient. The model included four major criteria: Prevent Cancer, Avoid Side Effects, Minimize False Positives, and Optimal Test Logistics. The latter criterion had three sub-criteria: screening frequency, preparation, and the test procedure. Linked elements among comparison sets were used to reduce the number of comparisons among the options from 187 to 76 . We used hierarchical cluster analysis to identify common sets of priorities for the major decision criteria.
\end{abstract}

Results: The study sample consists of 27 academic PCPs, 19 men and 8 women, mean age 41 years. All physicians completed comparisons of the major decision criteria; 21 completed the entire analysis. The median consistency ratios for the major criteria comparisons were 0.15 and 0.12 for the entire analysis. Hierarchical cluster analysis of the major criteria priorities revealed three discreet clusters with 10, 7, and

${ }^{*}$ Corresponding author 
10 members. Prevent Cancer was the most important criterion in every cluster. Each of the other criteria was the second most important priority in one cluster. Priority differences among clusters for all criteria were statistically significant $(\mathrm{P}<0.001)$.

Conclusion: PCPs' decision priorities regarding considerations affecting the choice among currently recommended CRC screening tests can be assessed using the AHP. While preventing cancer appears to be the most important consideration, several other factors play an important role in choosing a screening option.

Keywords: Medical decision making, colorectal cancer screening, linked elements

\section{Introduction}

Colorectal cancer (CRC) is the third most common cancer diagnosed in the United States and the second leading cause of cancer mortality. (American Cancer Society) Although there is general agreement that screening average risk patients for colorectal cancer is worthwhile, there is no similar consensus on how screening should be accomplished. Current United States CRC screening guidelines endorse several screening strategies and recommend that a strategy be selected based on an individualized assessment of the respective advantages and disadvantages. (Levin, et al., 2008; U.S. Preventive Services Task Force recommendation statement, 2008)

By presenting multiple options that have different combinations of strengths and weaknesses, current CRC screening guidelines present doctors and patients with a complex decision task. Cognitive science research has shown that people frequently have difficulty making consistently good decisions in situations like this. (Redelmeier \& Shafir, 1995; Russo, Carlson, \& Meloy, 2006; Ubel, 2002) These findings suggest a better understanding of how clinical decision makers think about the tradeoffs inherent in current CRC screening guidelines will improve the effectiveness of CRC prevention in clinical practice.

Most colorectal cancer screening decisions are made in primary care settings. Although current guidelines recommend that $\mathrm{CRC}$ screening decisions be made through a shared decision making process, physician recommendations greatly influence colorectal cancer screening choices for many patients. (Klabunde, et al., 2005; Seeff, et al., 2004) We therefore analyzed priorities assigned by primary care physicians using the Analytic Hierarchy Process (AHP) to the criteria that differentiate currently recommended CRC screening strategies.

\section{Methods}

\section{Study Population}

The study population consisted of 27 primary care physicians from Indianapolis IN and Rochester, NY who agreed to participate in a study of decision-making regarding colorectal cancer screening in primary care practice.

\section{The study intervention}

The study intervention consisted of a description of the study, a brief review of current (2006) colorectal cancer screening guidelines, collection of demographic and background information, and a multi-criteria analysis of the decision regarding the choice of a colorectal cancer screening strategy for a typical healthy, average risk 50 year old patient using the Analytic Hierarchy Process (AHP). (Saaty, 1980, 2001a; Saaty, 1990) The majority of physicians completed the study intervention at a large group meeting 
at each study site at the start of the study. Physicians who were unable to attend were subsequently interviewed individually.

\section{6 screening guidelines}

At the time of the study, screening guidelines for average risk patients included six options: annual guaiac-based fecal occult blood tests, annual immunochemical fecal occult blood tests, flexible sigmoidoscopy every five years, combined annual fecal occult blood tests and flexible sigmoidoscopy every five years, double-contrast barium enema every five years, and colonoscopy every ten years. For all non-colonoscopy options, a follow up colonoscopy was recommended if the initial test was positive. (Pignone, Rich, Teutsch, Berg, \& Lohr, 2002; S. Winawer, et al., 2003)

\section{Multi-criteria analysis using the Analytic Hierarchy Process}

The decision model used for the study is shown in Figure 1. The goal was to choose the "best" colorectal cancer screening program for a typical, average risk 50 year old patient. There were four major criteria: Prevent Cancer, effectiveness in preventing cancer morbidity and mortality; Avoid Side Effects, minimize the risk of serious screening-related side effects defined as intestinal perforation or bleeding severe enough to require hospitalization; Minimize False Positives, minimize the chance of a false positive screening test; and Optimal Test Logistics, the combined importance of three procedure-related characteristics that were included in the model as sub-criteria: Frequency, the frequency of screening; Preparation, the preparation required for screening; and Procedure, the nature of the screening procedure itself.

Figure 1. Decision Model Presented to Primary Care Physicians to Determine Best Clinical Strategy

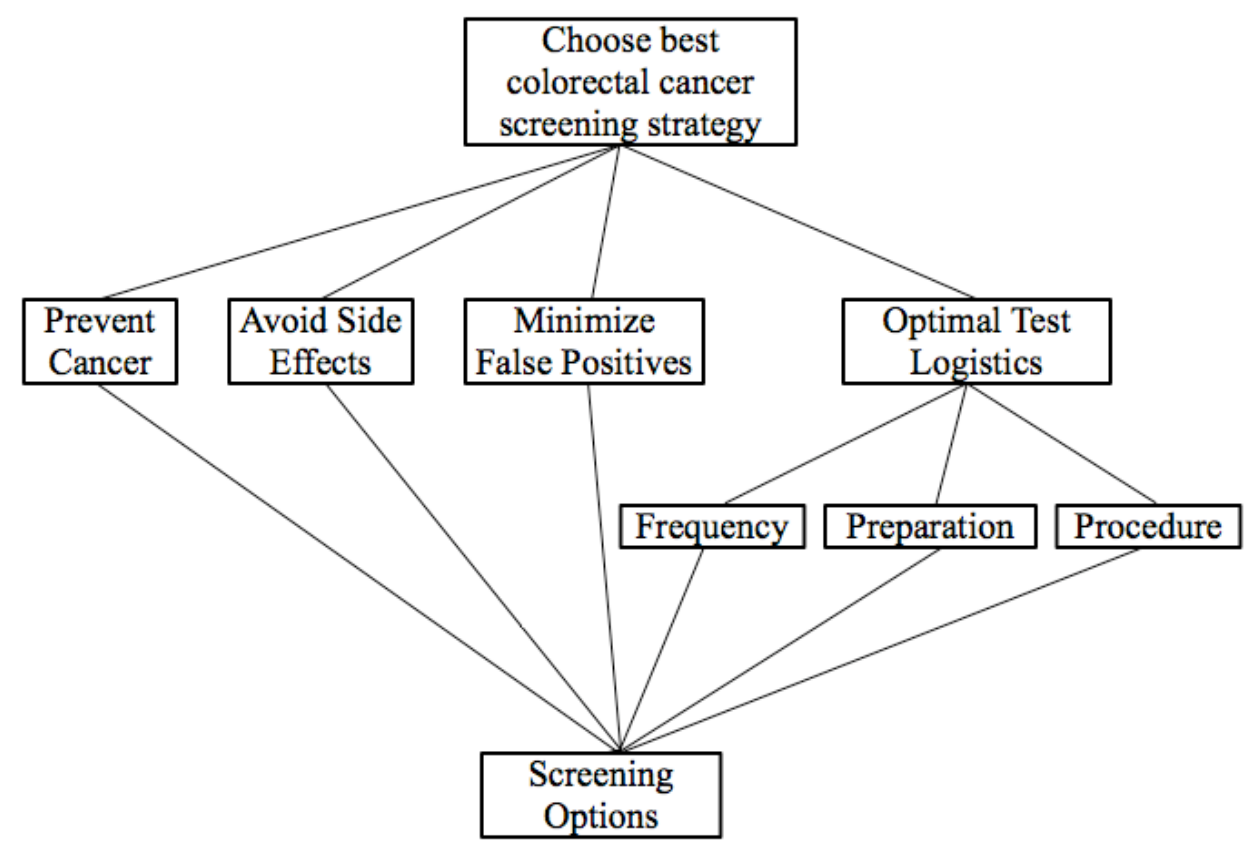

Ten screening options were included: the six recommended screening options listed previously and two additional tests that seemed likely to be included in future recommendations: Computer Tomographic 
Colonography (CTC) (also known as virtual colonoscopy) and fecal DNA testing. Because the reported sensitivity of non-rehydrated guaiac-based fecal occult blood tests varied widely, two guaiac options were included, with test sensitivities of $20 \%$ and $40 \%$ respectively. (Imperiale, et al., 2004; Lieberman, et al., 2001) Two combined fecal occult blood test and flexible sigmoidoscopy strategies were also included: one using guaiac-based fecal occult blood tests with $40 \%$ sensitivity and one using immunochemicalbased fecal occult blood tests.

The PCPs compared the alternatives with respect to Prevent Cancer, Avoid Side Effects, and Minimize False Positives using outcome estimates that assumed regular screening with the same program at the prescribed interval from age 50 through death or age 80 . These outcome estimates were obtained using current information about the sensitivity and specificity of screening tests and a modified version of a colorectal cancer simulation program originally developed for the first Multi-Disciplinary colorectal cancer screening guidelines that were published in 1997. (Miller, February 2006; Winawer, et al., 1997) The outcome estimates used for the study, and the other data used to make the comparisons, are summarized in Table 1. All comparisons, except those about the procedure, were done in a blinded fashion.

Table 1. Data used to compare colorectal cancer screening options.

\begin{tabular}{|l|l|l|l|l|l|}
\hline Screening option * & $\begin{array}{l}\text { Cancers } \\
\text { prevented per } \\
1,000\end{array}$ & $\begin{array}{l}\text { Serious side } \\
\text { effects } \\
100,000\end{array}$ & $\begin{array}{l}\text { False positive } \\
\text { tests per 1,000 }\end{array}$ & $\begin{array}{l}\text { Screening } \\
\text { frequency } \\
\text { (years) }\end{array}$ & Test preparation \\
\hline FOBT 20 & 11 & 104 & 706 & 1 & Diet \\
\hline FOBT 40 & 19 & 104 & 706 & 1 & Diet \\
\hline iFOBT & 27 & 91 & 476 & 1 & None \\
\hline FlexSig & 20 & 19 & 0 & 5 & Enema \\
\hline FOBT \& FlexSig & 29 & 114 & 706 & $1 \& 5$ & Diet \& enema \\
\hline iFOBT \& FlexSig & 34 & 99 & 486 & $1 \& 5$ & Diet \& enema \\
\hline fDNA & 23 & 178 & 1000 & 3 & None \\
\hline CT scan & 30 & 44 & 11 & 3 & Full colon prep \\
\hline DCBE & 33 & 46 & 10 & 5 & Full colon prep \\
\hline Colonoscopy & 36 & 326 & 0 & 10 & Full colon prep \\
\hline
\end{tabular}

* - Abbreviations: FOBT $=$ fecal occult blood test; iFOBT $=$ immunochemical fecal occult blood test; FlexSig = flexible sigmoidoscopy; fDNA = fecal DNA test; DCBE = double contrast barium enema

To reduce the number of comparisons needed for the analysis, linked elements were used to divide the ten alternatives into two groups of four and one group of three for the comparisons relative to Prevent Cancer, Avoid Side Effects, Minimize False Positives, and Procedure. A linked element is a common alternative contained in two comparison subsets that can be used to create a uniformly scaled, complete comparison matrix. (Saaty, 2001b) This procedure reduced the number of required comparisons for each of these criteria from the 45 pairwise comparisons needed to analyze a set of ten alternatives to the 15 needed to compare two sets of four alternatives ( 6 comparisons each) and one set of three alternatives ( 3 comparisons).

Comparisons among the five possible screening preparations and the four possible screening frequencies were made as single sets requiring ten and six comparisons, respectively. The total number of comparisons needed to compare the alternatives relative to the criteria was 76:15 comparisons for each of the four criteria with linked elements plus 16 for the two criteria compared without linked criteria. 
The study physicians completed the AHP analysis using a paper form. Preference intensities for both the alternative and criteria comparisons were measured by placing a mark on a horizontal line. Figure 2 illustrates this format.

Figure 2. The format used to make the AHP pairwise comparisons.

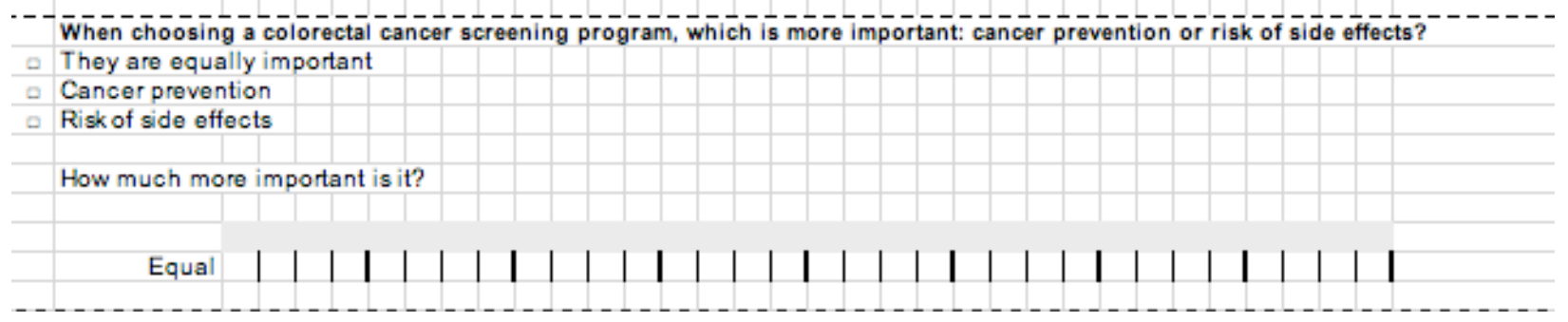

All priorities were calculated using the normalized right principal eigenvector procedure. Consistency ratios for each set of comparisons were calculated by first calculating the consistency index using the following formula:

$$
\mathrm{CI}=\left(\lambda_{\max }-\mathrm{n}\right) /(\mathrm{n}-1)
$$

where $\mathrm{CI}$ equals the consistency index, $\lambda_{\max }$ equals the largest eigenvalue of the comparison matrix, and $\mathrm{n}$ is the number of items being compared. We then calculated the consistency index by dividing the consistency index by the random index for the same size matrix. The random index is the mean consistency index for 500 randomly generated matrices. (Saaty, 1980) We calculated the consistency ratios for the overall analysis by multiplying the consistency ratio of each comparison set by the priority assigned to the criterion being addressed. We used this same weighted approach to calculate the consistency ratios for the linked sets of comparisons. We defined comparisons with consistency ratios $\leq$ 0.10 as consistent and those with consistency ratios between 0.11 and 0.21 as mostly consistent. (Forman \& Selly, 2001; Katsumura, Yasunaga, Imamura, Ohe, \& Oyama, 2008; Sato, 2004)

All AHP calculations were performed using a programmed Microsoft Excel spreadsheet. The results were validated using Expert Choice 2000, a commercially available AHP software program. (Expert Choice, Inc., Arlington VA)

\section{Cluster Analysis}

Cluster analysis refers to statistical procedures that identify groups of subjects with similar responses to a set of selected variables within a dataset. By helping organize and classify data, cluster analysis can help provide a better understanding of the information within a dataset. A hierarchical cluster analysis starts with each data point in its own cluster and then sequentially groups them together using a one of several standard algorithms.

We performed a hierarchical cluster analysis of the major criteria priorities provided by the study physicians using Ward's method as implemented in ClustanGraphics. (Wishart, 2006) Data regarding the priorities assigned to the four major decision criteria - Prevent Cancer, Avoid Side Effects, Minimize False Positives, and Optimize Test Logistics - were first converted to standardized z-scores and then clustered using the increase in the sum of squares of the squared Euclidean distance method.

\section{Data analysis}

We summarized the data using standard descriptive procedures and tested the statistical significance of overall priority differences within clusters using analysis of variance. The statistical significance of 
priority differences between specific clusters was examined using the Bonferroni-Dunn test. All statistical analyses were conducted using Aabel 3. (Aabel 3, Gigawiz Ltd. Co., 2008)

\section{Results}

The characteristics of the 27 study physicians are summarized in Table 2 .

Table 2. Characteristics of the study population.

\begin{tabular}{|l|c|}
\hline Number & 27 \\
\hline Age (mean, range) & $42.8(31-68)$ \\
\hline Years in practice (mean, standard deviation) & $12.5(10.2)$ \\
\hline Gender (n, percent) & $19(70 \%)$ \\
Male & $8(30 \%)$ \\
Female & \\
\hline Study site (n, percent) & $8(30 \%)$ \\
Rochester & $19(70 \%)$ \\
Indianapolis & \\
\hline
\end{tabular}

The results of the physicians' AHP analyses are summarized in Table 3. Prevent Cancer was the most important major criterion, with a mean priority of 0.524 . The mean priorities assigned to the other three major criteria were similar and ranged from 0.144 for Optimal Test Logistics to 0.175 for Avoid Side Effects. The mean and median consistency ratios for the major criteria comparisons were both 0.15 . Fourteen $(52 \%)$ of the 27 physicians had consistency ratios $\leq 0.10 ; 6(22 \%)$ had ratios $>0.20$.

Procedure was the most important of the subcriteria with a mean priority of 0.497 , twice as important as the other two subcriteria, Frequency (mean priority 0.256) and Preparation (mean priority 0.247). The mean and median consistency ratios for the sub-criteria priority comparisons were 0.16 and 0.05 . Twentytwo $(81 \%)$ of the 27 physicians had consistency ratios $\leq 0.10$.

Table 3. The primary care physicians' criteria priorities.

\begin{tabular}{|l|c|}
\hline Criterion Priority & Mean priority score (standard deviation) \\
\hline Major Criteria & $0.524(0.088)$ \\
\hline Prevent Cancer & $0.175(0.083)$ \\
\hline Avoid Side Effects & $0.155(0.143)$ \\
\hline Minimize False Positives & $0.145(0.083)$ \\
\hline Optimize Test Logistics & $0.15(0.11)$ \\
\hline Consistency ratio & \\
\hline Optimal Test Logistics sub-criteria & $0.256(0.152)$ \\
\hline Frequency priority & $0.247(0.125)$ \\
\hline Preparation priority & $0.497(0.166)$ \\
\hline Procedure priority & $0.156(0.3)$ \\
\hline Consistency ratio &
\end{tabular}

Twenty-one physicians successfully completed the full AHP analysis. The primary reason for incomplete analyses was failure to correctly fill out the paper response forms. The AHP-best alternative was colonoscopy every 10 years for $20(95 \%)$ physicians and flexible sigmoidoscopy every 5 years for one $(5 \%)$. The mean and median consistency ratios for the full analyses were both 0.12 . Fifteen $(71 \%)$ physicians had ratios $\leq 0.10$; two $(10 \%)$ were $>0.20$. 
The hierarchical cluster analysis of the major criteria priorities resulted in three clusters, containing 10, 7, and 10 physicians each. The details are provided in Table 4; the differences among the clusters are illustrated in Figure 3. Prevent Cancer was ranked the most important criterion in all three clusters. Each of the other three major criteria was ranked second most important in one cluster: Avoid Side Effects in cluster 3, Avoid False Positives in cluster 2, and Optimize Test Logistics in cluster 1.The differences in criteria rankings across the clusters were statistically significant for all four criteria. There were no significant among-cluster differences in consistency ratios for either the major criteria comparisons or the overall AHP analyses.

Table 4. Characteristics of the three clusters based on major criteria priorities.

\begin{tabular}{|c|c|c|c|c|}
\hline Criterion & $\begin{array}{c}\text { Cluster 1 } \\
(\mathrm{n}=10)\end{array}$ & $\begin{array}{c}\text { Cluster 2 } \\
(\mathrm{n}=7)\end{array}$ & $\begin{array}{c}\text { Cluster } 3 \\
(\mathrm{n}=10)\end{array}$ & \\
\hline & \multicolumn{3}{|c|}{ Mean priority (standard deviation) } & F ratio \\
\hline Prevent Cancer & $0.562(0.11)$ & $0.393(0.16)$ & $0.577(0.06)$ & $6.5, p=0.006,{ }^{2,3}$ \\
\hline Avoid Side Effects & $0.126(0.06)$ & $0.133(0.06)$ & $0.252(0.05)$ & $13.9, \mathrm{p}<0.001,1,3$ \\
\hline Minimize False Positives & $0.078(0.05)$ & $0.359(0.14)$ & $0.09(0.03)$ & $33.7, \mathrm{p}<0.001,,^{2,3}$ \\
\hline Optimize Test Logistics & $0.233(0.03)$ & $0.116(0.06)$ & $0.076(0.04)$ & $34.1, \mathrm{p}<0.001,{ }^{1,2}$ \\
\hline Consistency ratio, major criteria & $0.142(0.13)$ & $0.166(0.14)$ & $0.146(0.07)$ & $0.1, \mathrm{p}>0.05$ \\
\hline Consistency ratio, overall & $0.096(0.05)$ & $0.17(0.07)$ & $0.12(0.03)$ & $2.8, \mathrm{p}=0.085$ \\
\hline
\end{tabular}

1. Difference between clusters 1 and 2 statistically significant by Bonferroni-Dunn Test, $\mathrm{p}<0.001$.

2. Difference between clusters 1 and 3 statistically significant by Bonferroni-Dunn Test, $p<0.001$.

3. Difference between clusters 2 and 3 statistically significant by Bonferroni-Dunn Test, $p<0.001$.

Figure 3. Boxplot of the results of the cluster analysis of the major criteria priorities. The dark gray figures represent cluster 1, the light gray figures cluster 2 and the white figures cluster 3 . The boxes show 25 th -75 th interquartile range and the whiskers the 95 th percentiles. Means are denoted by the diamonds; medians by the horizontal lines..

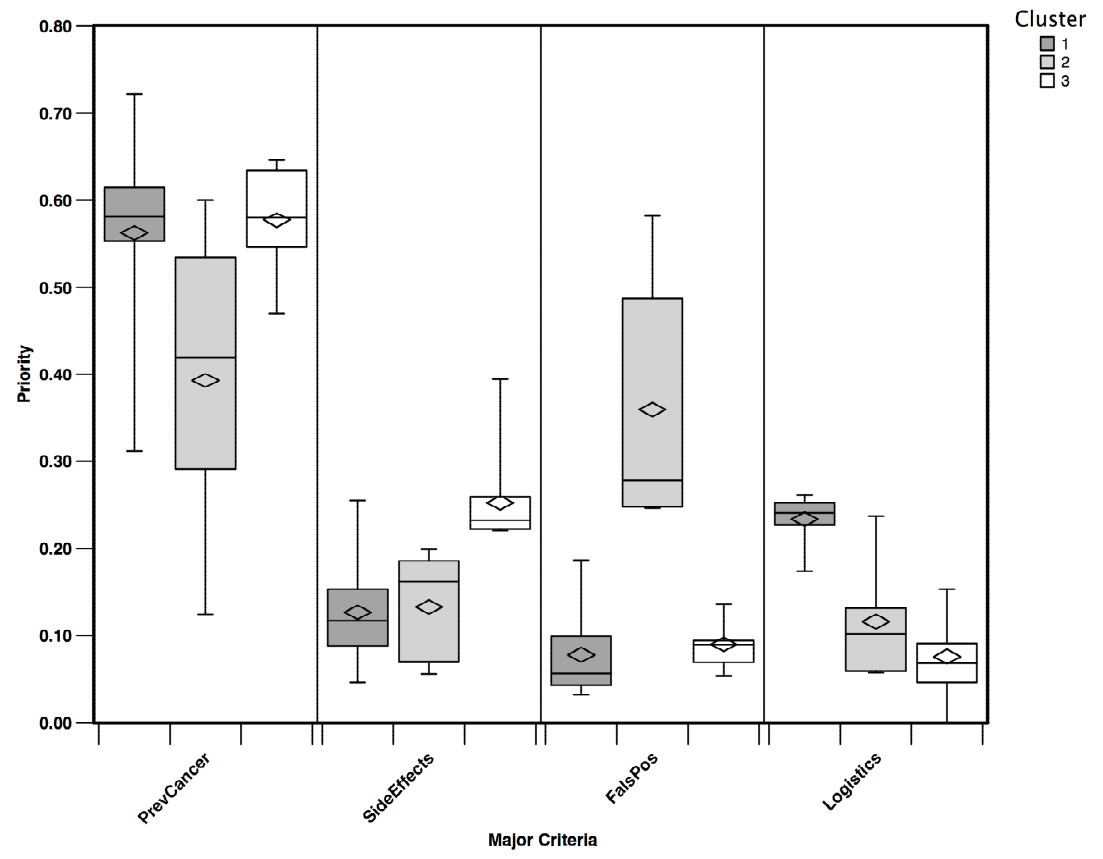


Table 5 summarizes the top five screening alternatives in each cluster. The same five screening options were ranked in each. Colonoscopy every 10 years was the highest ranked screening option in every cluster. The rankings of the other four options differed for each.

Table 5. The top five screening alternatives, by cluster

\begin{tabular}{|l|c|l|c|l|c|}
\hline \multicolumn{2}{|c|}{ Cluster 1 } & \multicolumn{2}{c|}{ Cluster 2 } & \multicolumn{2}{c|}{ Cluster 3 } \\
\hline Alternative & Score (\%) & Alternative & Score & Alternative & Score \\
\hline Cscope & 24.3 & Cscope & 30.2 & Cscope & 28.9 \\
\hline DCBE & 13.8 & FlexSig & 19.5 & FlexSig & 17.5 \\
\hline iFOBT \& FlexSig & 13.4 & DCBE & 14.0 & DCBE & 14.0 \\
\hline CT & 11.2 & CT & 10.8 & iFOBT \& FlexSig & 13.7 \\
\hline FlexSig & 10.8 & iFOBT \& FlexSig & 8.9 & CT & 11.2 \\
\hline
\end{tabular}

Abbreviations: Cscope $=$ colonoscopy every 10 years; $\mathrm{DCBE}=$ double contrast barium enema every 5 years; iFOBT \& FlexSig = annual immunochemical fecal occult blood tests and flexible sigmoidoscopy every 5 years; $\mathrm{CT}=\mathrm{CT}$ colonography; FlexSig $=$ flexible sigmoidoscopy every 5 years .

\section{Discussion}

In this study, we found that physician subjects learned how to use the AHP to examine colorectal screening decisions and prioritize decision criteria with minimal instruction. Most were also able to perform the necessary pairwise comparisons with acceptable levels of consistency and complete a very complex analysis despite the lack of any feedback during the process. These findings suggest that a clinical decision support system targeted at practicing physicians using the AHP is feasible and could be used to help assess and compare their decision-making priorities. To be successful, however, such systems will need to include provision of ongoing feedback during the analysis to avoid procedural mistakes such as those that occurred in this study.

Although all study physicians considered preventing cancer the most important criterion, as a group they assigned almost half of the overall decision priority to the other criteria. As shown by the differences among the three clusters, there was significant variability in how the study physicians viewed the relative importance of these three considerations. These results suggest that, when given the opportunity, primary care physicians view the choice of colorectal cancer screening strategy as a multi-criteria decision problem and vary in the how they assign decision priorities.

The results of the cluster analysis raise the possibility that primary care physicians prioritize these criteria in predictable patterns. This finding is similar to results of a previous study of patient colorectal cancer screening priorities that also found evidence of similar groups of common priority assessments. (Dolan, 2005) If confirmed, these findings suggest that rapid clinical support systems could be developed based on matching empirically derived sets of common patient and provider decision preferences.

Despite the variations found in decision priorities, colonoscopy every 10 years was the top-ranked screening option in all three clusters and for 20 of the 21 physicians. The clinical significance of this finding is uncertain however, since it is important to consider both physician and patient preferences when making colorectal cancer screening choices. Previous studies have consistently shown differences between doctors and patients in how they assess decision priorities. (Dolan, Bordley, \& Miller, 1993; Peralta-Carcelen, Fargason, Coston, \& Dolan, 1997) Additional studies comparing patient and physician priorities are needed to address this question. 
This study has two main limitations. The first is that the study population consisted of a small, sample of physicians consisting almost entirely of academic general internists. The extent to which the study findings generalize to other primary care providers is unknown. The second is that the stability of the decision priorities over time was not evaluated.

Despite these limitations, we conclude that this study provides evidence that primary care physicians are capable of using the AHP to analyze a complex problem regarding selection of a colorectal cancer screening option and that they consider multiple considerations important when making this decision. Additional research is warranted to confirm these findings and to determine the extent to which patient priorities and preferred screening alternatives agree with those of their primary care physicians. 


\section{References}

Aabel 3, Gigawiz Ltd. Co. (2008).

American Cancer Society. Cancer Facts \& Figures 2008 Graphs and Figures, from http://www.cancer.org/docroot/MED/content/MED_1_1_Most_Requested_Graphs_and_Figures_2008.as $\mathrm{p}$

Dolan, J. (2005). Patient priorities in colorectal cancer screening decisions. Health Expect, 8(4), 334-344.

Dolan, J. G., Bordley, D. R., \& Miller, H. (1993). Diagnostic strategies in the management of acute upper gastrointestinal bleeding: patient and physician preferences. J Gen Intern Med, 8(10), 525-529.

Expert Choice 2000. Expert Choice, Inc. (2000).

Forman, E., \& Selly, M. (2001). Decision by objectives. Hackensack, NJ: World Scientific.

Imperiale, T. F., Ransohoff, D. F., Itzkowitz, S. H., Turnbull, B. A., Ross, M. E., \& the Colorectal Cancer Study Group (2004). Fecal DNA versus Fecal Occult Blood for Colorectal-Cancer Screening in an Average-Risk Population. N Engl J Med, 351(26), 2704-2714.

Katsumura, Y., Yasunaga, H., Imamura, T., Ohe, K., \& Oyama, H. (2008). Relationship between risk information on total colonoscopy and patient preferences for colorectal cancer screening options: Analysis using the Analytic Hierarchy Process. BMC Health Services Research, 8(1), 106-106.

Klabunde, C. N., Vernon, S. W., Nadel, M. R., Breen, N., Seeff, L. C., \& Brown, M. L. (2005). Barriers to colorectal cancer screening: a comparison of reports from primary care physicians and average-risk adults. Medical Care, 43(9), 939-944.

Levin, B., Lieberman, D. A., McFarland, B., Smith, R. A., Brooks, D., Andrews, K. S., et al. (2008). Screening and surveillance for the early detection of colorectal cancer and adenomatous polyps, 2008: a joint guideline from the American Cancer Society, the US Multi-Society Task Force on Colorectal Cancer, and the American College of Radiology. CA Cancer J Clin, 58(3), 130-160.

Lieberman, D. A., Harford, W. V., Ahnen, D. J., Provenzale, D., Sontag, S. J., Schnell, T. G., et al. (2001). One-Time Screening for Colorectal Cancer with Combined Fecal Occult-Blood Testing and Examination of the Distal Colon. N Engl J Med, 345(8), 555-560.

Miller, L. Personal communication, February 2006.

Peralta-Carcelen, M., Fargason, C. A., Jr., Coston, D., \& Dolan, J. G. (1997). Preferences of pregnant women and physicians for 2 strategies for prevention of early-onset group B streptococcal sepsis in neonates. Arch Pediatr Adolesc Med, 151(7), 712-718.

Pignone, M., Rich, M., Teutsch, S., Berg, A., \& Lohr, K. (2002). Screening for colorectal cancer in adults at average risk: A summary of the evidence for the U.S. Preventive Services Task Force. Ann Intern Med, $137(2), 132-141$. 
Redelmeier, D., \& Shafir, E. (1995). Medical decision making in situations that offer multiple alternatives. JAMA, 273(4), 302-305.

Russo, J. E., Carlson, K. A., \& Meloy, M. G. (2006). Choosing an Inferior Alternative. Psychological Science, 17(10), 899-904.

Saaty, T. (1980). The Analytic Hierarchy Process. New York: McGraw-Hill Book Co.

Saaty, T. (2001a). Decision Making for Leaders (3rd ed.). Pittsburgh, PA: RWS Publications.

Saaty, T. (2001b). The Analytic Network Process: Decision Making With Dependence and Feedback. Pittsburgh: RWS Publications.

Saaty, T. L. (1990). How to make a decision: The analytic hierarchy process. European Journal of Operational Research, 48(1), 9-26.

Sato, J. (2004). Comparison between multiple-choice and analytic hierarchy process: measuring human perception. International Transactions in Operational Research, 11(1), 77-86.

Screening for colorectal cancer: U.S. Preventive Services Task Force recommendation statement (2008). Ann Intern Med, 149(9), 627-637.

Seeff, L. C., Nadel, M. R., Klabunde, C. N., Thompson, T., Shapiro, J. A., Vernon, S. W., et al. (2004). Patterns and predictors of colorectal cancer test use in the adult U.S. population. Cancer, 100(10), 20932103.

Ubel, P. A. (2002). Is information always a good thing? Helping patients make "good" decisions. Med Care, 40(9 Suppl), V39-44.

Winawer, S., Fletcher, R., Rex, D., Bond, J., Burt, R., \& al, e. (2003). Colorectal cancer screening and surveillance: Clinical guidelines and rationale - Update based on new evidence. Gastroenterology, 124, 544-560.

Winawer, S. J., Fletcher, R. H., Miller, L., Godlee, F., Stolar, M. H., Mulrow, C. D., et al. (1997). Colorectal cancer screening: clinical guidelines and rationale. Gastroenterology, 112(2), 594-642.

Wishart, D. (2006). ClustanGraphics (Version fourth edition). Edinburgh: Clustan Limited. 